\title{
Wrist-worn accelerometers in assessment of energy expenditure during intensive training
}

Citation for published version (APA):

Kinnunen, H., Tanskanen, M., Kyrolainen, H., \& Westerterp, K. R. (2012). Wrist-worn accelerometers in assessment of energy expenditure during intensive training. Physiological Measurement, 33(11), 18411854. https://doi.org/10.1088/0967-3334/33/11/1841

Document status and date:

Published: 01/01/2012

DOI:

10.1088/0967-3334/33/11/1841

Document Version:

Publisher's PDF, also known as Version of record

Document license:

Taverne

Please check the document version of this publication:

- A submitted manuscript is the version of the article upon submission and before peer-review. There can be important differences between the submitted version and the official published version of record.

People interested in the research are advised to contact the author for the final version of the publication, or visit the DOI to the publisher's website.

- The final author version and the galley proof are versions of the publication after peer review.

- The final published version features the final layout of the paper including the volume, issue and page numbers.

Link to publication

\footnotetext{
General rights rights.

- You may freely distribute the URL identifying the publication in the public portal. please follow below link for the End User Agreement:

www.umlib.nl/taverne-license

Take down policy

If you believe that this document breaches copyright please contact us at:

repository@maastrichtuniversity.nl

providing details and we will investigate your claim.
}

Copyright and moral rights for the publications made accessible in the public portal are retained by the authors and/or other copyright owners and it is a condition of accessing publications that users recognise and abide by the legal requirements associated with these

- Users may download and print one copy of any publication from the public portal for the purpose of private study or research.

- You may not further distribute the material or use it for any profit-making activity or commercial gain

If the publication is distributed under the terms of Article $25 \mathrm{fa}$ of the Dutch Copyright Act, indicated by the "Taverne" license above, 


\section{PAPER}

\section{Wrist-worn accelerometers in assessment of energy expenditure during intensive training}

To cite this article: H Kinnunen et al 2012 Physiol. Meas. 331841

View the article online for updates and enhancements.

\section{Recent citations}

$$
\begin{aligned}
& \text { - Association between accelerometer- } \\
& \frac{\text { measured physical activity, alucose }}{\text { metabolism, and waist circumference in }} \\
& \frac{\text { older adults }}{\text { Länsitie Miia et al }} \\
& \text { - Soldiers' physical activity of daily life: a } \\
& \frac{\text { svstematic literature review }}{\text { Christin Schilz and Stefan Sammito }} \\
& \text { - Negative energy balance during military } \\
& \frac{\text { training: the role of contextual limitations }}{\text { Keyne Charlot }}
\end{aligned}
$$

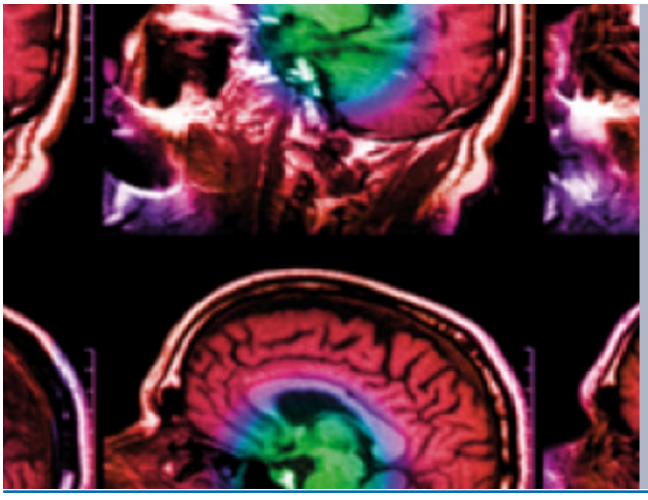

\section{IPEM IOP}

Series in Physics and Engineering in Medicine and Biology

Your publishing choice in medical physics, biomedical engineering and related subjects. Start exploring the collection-download the first chapter of every title for free. 


\title{
Wrist-worn accelerometers in assessment of energy expenditure during intensive training
}

\author{
H Kinnunen ${ }^{1,2}$, M Tanskanen ${ }^{3}$, H Kyröläinen ${ }^{3}$ and K R Westerterp ${ }^{4}$ \\ ${ }^{1}$ Polar Electro Oy, Kempele, Finland \\ ${ }^{2}$ Department of Electrical Engineering, University of Oulu, Finland \\ ${ }^{3}$ Department of Biology of Physical Activity, University of Jyväskylä, Finland \\ ${ }^{4}$ Department of Human Biology, Maastricht University, Maastricht, The Netherlands \\ E-mail: hannu.kinnunen@polar.fi
}

Received 27 April 2012, accepted for publication 12 September 2012

Published 31 October 2012

Online at stacks.iop.org/PM/33/1841

\begin{abstract}
We assessed the ability of the Polar activity recorder (AR) to measure energy expenditure (EE) during military training. Twenty-four voluntary male conscripts participated in the study and wore an AR on the non-dominant wrist $24 \mathrm{~h}$ a day for $7 \mathrm{~d}$. The AR analyzed and stored the frequency of hand movements ( $f_{-}$hand) into memory at 1 min intervals. The relationship between $f \_$hand and EE was studied over a $7 \mathrm{~d}$ period of military training using the doubly labeled water (DLW) technique. In addition, the relationship between $f$ hand and EE was analyzed during walking and running on a treadmill with an indirect calorimeter (IC), and $f_{-}$hand was measured during a supervised 45 min field march test where the conscripts carried combat gear. EE was expressed as physical activity level (PAL), total energy expenditure (TEE), and activity-induced energy expenditure adjusted for body mass (AEE/BM). Over the $7 \mathrm{~d}$ period, $f$ hand alone explained $46 \%$ of inter-individual variation in

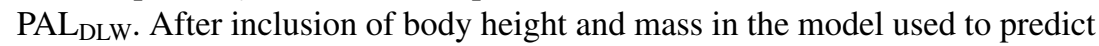
PAL DLW from $f \_$hand, a very high positive correlation and a low standard error of estimate (SEE) were observed between the AR and DLW techniques: for TEE $r=0.86(p<0.001)$, the SEE was $6.3 \%$, and for AEE/BM $r=0.84$ $(p<0.001)$, the SEE was $12.8 \%$. In the treadmill exercise, $f$ hand correlated highly with $\mathrm{PAL}_{\mathrm{IC}}(r=0.97 \pm 0.02)$. In the 45 min field march test, the AR measured similar $f$ hand as on the treadmill at the same speed. In conclusion, the wrist-worn AR can be regarded as a reliable and valid method for assessing EE during intensive training.
\end{abstract}

Keywords: accelerometers, doubly labeled water, energy expenditure, military training, physical activity

(Some figures may appear in colour only in the online journal) 


\section{Introduction}

Military service includes physically demanding tasks both indoors and outdoors and involves carrying loads and using various pieces of equipment and tools. The range of fitness levels among conscripts has widened (Santtila et al 2006), and the ability to tolerate intensive military training may be affected by increased body mass (BM) and lower fitness level (Tanskanen et al 2009). Subsequently, it would be important to have practical and reliable tools to monitor physical activity and energy expenditure (EE) in soldiers. During heavy training periods in the military and among endurance athletes, knowledge of EE could help in adjusting the training volume as well as controlling adequate energy availability. In order to be acceptable for the military, the methods need to be robust and unobtrusive.

EE is an important training parameter, since many measures of training load are associated with total energy expenditure (TEE). Physical activity level (PAL), defined as TEE divided by basal metabolic rate (BMR), has been used to classify occupational workload and leisure-time physical activity. PAL among North American and European adult population is typically 1.6-1.7 (Westerterp and Speakman 2008, Speakman and Westerterp 2010). An upper limit of PAL that can be maintained for a longer period of time has been determined to be about 2.5 within the general population (Black et al 1996, Westerterp and Plasqui 2004) while professional endurance athletes may reach higher PAL values (Westerterp 1998). Another measure for describing EE is activity-induced energy expenditure (AEE) with corrections for differences in body size (Ekelund et al 2004). AEE arises from muscular activity, including shivering and fidgeting as well as purposeful physical exercise (Poehlman 1989), and it is affected by the intensity and duration of activity as well as by individual differences in movement efficiency (Ainsworth et al 1993).

The doubly labeled water (DLW) technique is considered the golden standard for measuring EE under free-living conditions. Since the DLW technique is expensive, it is most often used in small study populations. While this technique provides an accurate measure of TEE, it gives no information on physical activity in terms of frequency, duration, and momentary intensity. Nevertheless, it is the only method available for accurately measuring TEE under free-living conditions (Plasqui and Westerterp 2007). In short-term measurements, indirect calorimetry (IC) has been extensively used as the reference method for assessing the validity of accelerometers in various sports and non-sports activities.

An accelerometer is the small instrument designed to register movement data at a high frequency over days or weeks. Accelerometers are typically uniaxial or triaxial, of which the latter are generally accepted as providing more information and a better relationship to AEE than uniaxial ones (Bouten et al 1994). Previously validated accelerometer applications (CSA/MTI/Actigraph and Tracmor) are worn close to the center of the body (hip, waist, or lower back), and they are designed to integrate the total amount of acceleration in one or three dimensions (Plasqui and Westerterp 2007). In contrast to these devices, Polar activity recorders (AR) utilize frequency and regularity of hand movements with adjustment for body parameters (Kinnunen et al 2009). This method requires little computational power, which has enabled its use in battery-operated wrist-worn devices (Polar AW200, FA20 and Active, Polar Electro Oy, Kempele, Finland).

Wrist-worn motion sensors were introduced in a research setting already in the 1950s, but until a recent study by van Hees et al (2011), there have been no validation data available from wrist-worn accelerometers with respect to TEE under free-living conditions (Plasqui and Westerterp 2007). Van Hees et al (2011) used a linear model to analyze the role of wrist acceleration in explaining variations in AEE, and they found that a wrist-worn threedimensional accelerometer explained $24 \%$ of variation in AEE in 73 non-pregnant women. 
In short-term recordings, activity monitoring from the wrist has been found to be accurate in measuring EE while walking and hiking (Bouten et al 1997, Brugniaux et al 2010). In daily tasks and sports activities, a minimal prediction improvement has been reported when a wrist accelerometer has been added to the center of body measurement (Swartz et al 2000, Tanaka et al 2007). However, Kinnunen et al (2009) have reported a reasonably high accuracy with the present methodology. Modern signal processing technology has allowed more complex analysis methods to improve the accuracy of accelerometer applications. For example, automatic activity classification has been widely studied in recent years with machine learning procedures or hierarchical algorithm structures, among other methods (Siirtola et al 2009, Yang and Hsu 2010, Zhang et al 2012).

In normal walking and running, the frequency of hand movement ( $f \_$hand) is linked with stride frequency (Wagenaar and van Emmerik 2000), and stride frequency is associated with walking and running speeds. Wixted et al (2007) described an EE estimator in walking and running based on step frequency modified by anthropometric measures. Anthropometrics such as leg length, body height $(\mathrm{BH})$ and $\mathrm{BM}$ affect self-selected step length and stride frequency at a given speed. Walking cadence has also been successfully used to determine the lower limit for moderate-intensity activity (Tudor-Locke and Rowe 2012). Another recent paper by Cheng et al (2009) describes an algorithm for estimation of exercise rate, i.e. stride rate in walking or running, pedaling cadence in cycling or stroke rate in rowing. All of these approaches have used waist mounted accelerometers, and apparently none of them has been validated in the long-term field setting so far.

The aim of this study was to assess the validity and reliability of the wrist-worn AR in measuring EE in a military environment. Because of usability issues and extreme measurement conditions, simple and robust methods were preferred. Firstly, a piece-wise linear relationship between $f$ hand and intensity of exercise was determined so that the best match was attained with AR and DLW regarding TEE and AEE during $7 \mathrm{~d}$ of intensive military training. Secondly, the relationship between $f \_$hand and intensity of exercise was determined with IC in treadmill walking and running on a minute-by-minute basis. Finally, the effect of carrying $20 \mathrm{~kg}$ of extra military equipment on $f$ hand was studied when walking or running at a constant speed on flat terrain covered by fine gravel.

\section{Methods}

\subsection{Subjects}

Twenty-four voluntary male conscripts, age 19-20 years, mean \pm SD BM $77 \pm 15$ (range 57-111) $\mathrm{kg}, \mathrm{BH} 178 \pm 8(153-187) \mathrm{cm}$, and maximal oxygen uptake $\left(\mathrm{VO}_{2 \max }\right) 47 \pm 6$ (30-59) $\mathrm{ml} \mathrm{kg}^{-1} \mathrm{~min}^{-1}$, participated in the study. The selection of subjects from a group of voluntary subjects is explained in detail by Tanskanen et al (2009). The subjects' physical fitness characteristics were representative of young Finnish men (Santtila et al 2006). All the subjects were informed of the experimental protocol and they gave their written consent to participate in the study. They were also advised of their right to withdraw from the investigation at any time. The study protocol was approved by the Finnish Defense Forces and the Ethics Committees of the University of Jyväskylä and the Kainuu region.

\subsection{Study protocol}

The experimental protocol, timing of the tests, and main daily program with respect to the DLW assessment period, are illustrated in table 1 . The study protocol included a treadmill 
Table 1. Experimental protocol and main daily program during the DLW assessment period.

\begin{tabular}{|c|c|c|c|c|c|c|c|c|c|c|c|}
\hline & \multicolumn{11}{|c|}{ Days } \\
\hline & $-9, \ldots,-5$ & 0 & 1 & 2 & 3 & 4 & 5 & 6 & 7 & 8 & +1 \\
\hline $\mathrm{VO}_{2 \max }$ test on a treadmill & $x$ & & & & & & & & & & \\
\hline $\begin{array}{l}\text { DLW (doubly labeled water) } \\
\text { dose }\end{array}$ & & $x$ & & & & & & & & & \\
\hline Urine sample & & $x$ & $x$ & & & & & & & $x$ & \\
\hline Combat shooting exercise & & $x$ & $\times$ & & & & & & & & \\
\hline Overnight field exercise & & & & $x$ & $x$ & $\times$ & $x$ & & & & \\
\hline Long-distance skiing & & & & & & & & & $\times$ & & \\
\hline Shooting exercise & & & & & & & & & & $x$ & \\
\hline 45 min march test & & & & & & & & & & & $x$ \\
\hline
\end{tabular}

test about 1 week before the DLW assessment period, and a 45 min field march test on the following day after the DLW period. The data collection took place during the winter when the ambient temperature varied between -2 and $-23{ }^{\circ} \mathrm{C}$.

\subsection{Polar AR}

Each conscript wore an AR (prototype of Polar FA20, Polar Electro Oy, Kempele, Finland) on the non-dominant wrist $24 \mathrm{~h}$ a day. As the sensing element, the AR included a capacitive 1D accelerometer. In the signal processing, the AR utilized a patented movement counting and filtering procedure (Kinnunen and Miettinen 2005). The acceleration signal was band-pass filtered $(0.3-3.0 \mathrm{~Hz})$ and the device counted hand movements if acceleration exceeded a preset threshold. The device did not register hand movements that appeared too soon after the previously detected movement, and the rejection time was longer in non-rhythmic activities. The number of registered hand movements was stored in the memory of the device at $1 \mathrm{~min}$ intervals, and this is referred to as $f \_$hand $\mathrm{AR}_{\mathrm{AR}}$. The prototype had a $24 \mathrm{~h}$ running memory, so the AR were collected every evening for data download and redistributed within $30 \mathrm{~min}$. If the AR had registered 30 consecutive zeros during the day (between 8 a.m. and 9 p.m.) and 300 consecutive zeros during the night (between 9 p.m. and 8 a.m.), the corresponding time was considered to be non-wear and left out of the analysis.

\subsection{Military training and DLW measurement}

Description of the $7 d$ military training. The measurement period was one of the most intensive training weeks during 8 week basic military training. The conscripts were involved in typical physically demanding military tasks, such as marching, material handling, and shooting exercises, and in several unsupervised activities of varying intensity in the garrison area and in the field. During the $7 \mathrm{~d}$ measurement period, the main events included a $1 \mathrm{~d}$ combat shooting exercise, $4 \mathrm{~d}$ of overnight field training, and $1 \mathrm{~d}$ of long distance skiing (table 1).

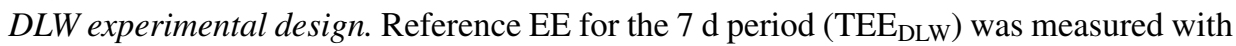
DLW according to the Maastricht Protocol (Westerterp et al 1995). Briefly, at 10 p.m. on day 0 after collecting a baseline urine sample, the subjects drank a weighed mixture of ${ }^{2} \mathrm{H}_{2} \mathrm{O}$ (99.9 atom\%) and $\mathrm{H}_{2}{ }^{18} \mathrm{O}$ (10 atom\%), resulting in an initial excess total body water enrichment of $150 \mathrm{ppm}$ for deuterium and $300 \mathrm{ppm}$ for oxygen-18. Total body water was estimated from calculated body composition based on BH, BM, age, and gender with the equation from Deurenberg et al (1991), assuming $73 \%$ hydration of fat-free mass. The subjects consumed no foods or fluids for $10 \mathrm{~h}$ after dose administration during overnight equilibration of the isotopes 
with body water. Subsequent urine samples were collected from the second and third voiding on the morning of day 1 , and from the first and second voiding on the morning of day 8 . Isotope quantities (deuterium and oxygen-18) in the urine were measured with an isotope ratio mass spectrometer (Optima, VG Isogas, Middlewich, $\mathrm{UK}$ ), and $\mathrm{CO}_{2}$ production was calculated from isotope ratios at the baseline and days 1 and 8 using the equations from Schoeller et al (1986). $\mathrm{CO}_{2}$ production was converted to daily metabolic rate using an energy equivalent based on the individual macronutrient composition of the diet (Black et al 1986). AEE $\mathrm{DLW}_{\mathrm{D}}$ was calculated as $\mathrm{TEE}_{\mathrm{DLW}} \times 0.9-\mathrm{BMR}$, assuming diet-induced thermogenesis of $10 \%$ (Poehlman 1989).

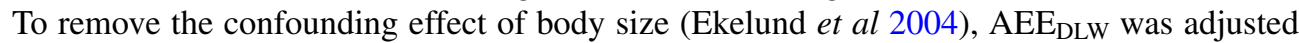
for $\mathrm{BM}\left(\mathrm{AEE} / \mathrm{BM}_{\mathrm{DLW}}\right)$. BM was measured on days 1 and 8 , and the average of these two measurements was used as individual BM.

Sick leave, substitution of lost AR data. Sick leave was non-attendance in daily service because of illnesses or injuries examined by a physician. Three of the subjects had a maximum of a $24 \mathrm{~h}$ period when they did not wear the AR because of sick leave. During that time their wrist activity counts were set to a very low level in the daytime (corresponding to PAL $=$ 1.6), and to zero activity at night (10 p.m.-7 a.m.). Other non-wear time was excluded from further analysis and we assumed that the individual average PAL of the analyzed wear time represented the entire $7 \mathrm{~d}$ period. In order to be included in further analysis, the subjects had to have successfully recorded AR data from a minimum of $4 \mathrm{~d}$.

\subsection{Treadmill test}

To determine $\mathrm{VO}_{2 \max }$ and to observe the relationship between $f$ hand $\mathrm{AR}_{\mathrm{R}}$ and the intensity of exercise in treadmill walking and running, the conscripts performed a maximal treadmill test. The test was performed in 3 min stages starting by walking at $4.6 \mathrm{~km} \mathrm{~h}^{-1}$ (1.0 inclination). Thereafter, treadmill speed and inclination were increased gradually every 3 min to induce an increase in intensity up to maximal effort. The procedure was the following: second stage: $6.3 \mathrm{~km} \mathrm{~h}^{-1}\left(1.0^{\circ}\right)$, third stage: $7.7 \mathrm{~km} \mathrm{~h}^{-1}\left(1.4^{\circ}\right)$, fourth stage: $9.0 \mathrm{~km} \mathrm{~h}^{-1}\left(1.9^{\circ}\right)$, fifth stage: $10.3 \mathrm{~km} \mathrm{~h}^{-1}\left(2.3^{\circ}\right)$, sixth stage: $11.3 \mathrm{~km} \mathrm{~h}^{-1}\left(3^{\circ}\right)$, seventh stage: $12.6 \mathrm{~km} \mathrm{~h}^{-1}\left(3.2^{\circ}\right)$, and eighth stage: $13.2 \mathrm{~km} \mathrm{~h}^{-1}\left(4.2^{\circ}\right)$. Pulmonary ventilation and respiratory gas exchange data were measured online by using the breath-by-breath method (Jaeger Oxygen Pro, VIASYS Healthcare GmbH, Hoechberg, Germany), and mean values were calculated at 1 min intervals for later analysis. Heart rate was continuously recorded at $5 \mathrm{~s}$ intervals using a heart rate monitor (Polar810i, Polar Electro Oy, Kempele, Finland). Blood lactate was determined 1 min after completion of exercise from a fingertip blood sample using a lactate analyzer (LactatePro ${ }^{\circledR}$, Arkray, Japan). The criteria used to determine maximal effort were: $\mathrm{VO}_{2}$ and heart rate did not increase despite an increase in inclination and speed of the treadmill, a respiratory exchange ratio higher than 1.1 , and a post-exercise blood lactate higher than $8 \mathrm{mmol}^{-1}$ (ASCM 2001).

Median $f_{-}$hand $\mathrm{AR}_{\mathrm{AR}}$ from each $3 \mathrm{~min}$ stage, as well as oxygen consumption $\left(\mathrm{VO}_{2}\right)$ and carbon dioxide production $\left(\mathrm{VCO}_{2}\right)$ from the final minute of each stage were extracted from the measured data. Only those 3 min stages that the subjects were able to finish were included in the analysis. A reference value $\left(\mathrm{PAL}_{\mathrm{IC}}\right)$ was calculated from respiratory gases and $\mathrm{BM}$ as follows: $\mathrm{EE}=3.8149 * \mathrm{O}_{2}(\mathrm{l})+1.2321 * \mathrm{CO}_{2}(\mathrm{l})($ Lusk 1924), $\mathrm{BMR}=15.1 * \mathrm{BM}+692$ (Schofield 1985), and finally, $\mathrm{PAL}_{\mathrm{IC}}=\mathrm{EE} / \mathrm{BMR}$.

\subsection{Field march test}

To assess the validity of the AR in quantifying the intensity of a typical military activity, $f \_$hand ${ }_{\mathrm{AR}}$ was determined during a 45 min field march test while the subjects carried a total 
of $20 \mathrm{~kg}$ of military equipment. The subjects marched in small groups at a constant speed in an indoor hall on a fine gravel surface. The speed was individually selected for each subject to match about $70 \%$ of measured $\mathrm{VO}_{2 \max }$. Five subjects marched at $6 \mathrm{~km} \mathrm{~h}^{-1}, 12$ at $7 \mathrm{~km} \mathrm{~h}^{-1}$, and 2 subjects at $8 \mathrm{~km} \mathrm{~h}^{-1}$. Constant and precise speed was ensured by a group leader who monitored the speed using a Polar S625X training computer (Polar Electro Oy, Kempele, Finland) equipped with an individually calibrated foot pod. The average $f$ hand AR $_{\text {measured }}$ during the march test was compared with the $f_{-}$hand $\mathrm{AR}_{\mathrm{A}}$ value measured during treadmill walking and running at the same speed. The average frequency was calculated over the 45 min march excluding 2 min from the beginning and the end. Because the speeds on the treadmill were not exactly the same as during marching, the treadmill data were individually interpolated between two treadmill stages to achieve $f_{-}$hand $\mathrm{AR}_{\mathrm{R}}$ that would best correspond to the same speed.

\subsection{Statistical analysis}

Customized model between the AR and PAL. Post-processing of the measured AR data was performed using Matlab software (The Mathworks Inc., Natick, MA). In order to derive EE parameters from the AR data, the first step was to model a piece-wise linear relationship between $f_{-}$hand $_{\mathrm{AR}}$ and PAL. Four pairs of $f \_$hand ${ }_{\mathrm{AR}}$ and PAL were determined: two intermediate points and the extreme values of $f \_$hand $d_{\mathrm{AR}}\left(0\right.$ and 100 movements $\left.\mathrm{min}^{-1}\right)$. As the second step, two per-cent-wise factors were determined to adjust PAL based on differences in $\mathrm{BH}$ and BM compared with the group mean values. The model parameters were selected so that the model would yield a best fit between the AR and DLW over the $7 \mathrm{~d}$ period. PAL $\mathrm{AR}_{\mathrm{A}}$ was determined as the average of the minute-by-minute values over the $7 \mathrm{~d}$ period. TEE $\mathrm{AR}_{\mathrm{A}}$ was calculated as $\mathrm{PAL}_{\mathrm{AR}} \times \mathrm{BMR}$, and $\mathrm{AEE} / \mathrm{BM}_{\mathrm{AR}}$ as $\left(\mathrm{TEE}_{\mathrm{AR}} \times 0.9-\mathrm{BMR}\right) / \mathrm{BM}$. Minimal 95\% limits of agreement between $\mathrm{TEE}_{\mathrm{AR}}$ and $\mathrm{TEE}_{\mathrm{DLW}}$ and a lack of trend in a Bland-Altman plot (Bland and Altman 1986) were used as the criteria of the best model.

Linear regression models. The statistical analyses were performed using PASW Statistics, version 18.0.0 (SPSS Inc). A Shapiro-Wilk test was used to verify that the data were normally distributed. In order to allow comparison with earlier studies and to identify the predictors of PAL ${ }_{\mathrm{DLW}}, \mathrm{TEE}_{\mathrm{DLW}}$, and $\mathrm{AEE} / \mathrm{BM}_{\mathrm{DLW}}$ in the $7 \mathrm{~d}$ assessment, stepwise multivariate linear regression analysis was used with $f_{-}$hand $\mathrm{AR}_{\mathrm{AR}}, \mathrm{BH}$, and $\mathrm{BM}$ as independent variables. In order to account for the effects of nonlinearity observed between $f$ hand ${ }_{\mathrm{AR}}$ and PAL, minute-byminute $f_{-}$hand $\mathrm{A}_{\mathrm{AR}}$ values were normalized based on the best piece-wise linear model so that the original range between 0 and 100 was maintained. The average normalized frequency of hand movements for the whole $7 \mathrm{~d}$ period is later referred to as $f$ hand_ $n_{\mathrm{AR}}$.

A paired-samples $t$-test was used to compare the measures of the $\mathrm{AR}\left(\mathrm{PAL}_{\mathrm{AR}}, \mathrm{TEE}_{\mathrm{AR}}\right.$, $\mathrm{AEE} / \mathrm{BM}_{\mathrm{AR}}$ ) with the reference DLW values (PAL ${ }_{\mathrm{DLW}}, \mathrm{TEE}_{\mathrm{DLW}}, \mathrm{AEE} \mathrm{DLW}_{\mathrm{W}}$ ) and to compare $f_{-}$hand $_{\mathrm{AR}}$ between the march and treadmill tests. Pearson correlation coefficients were computed to determine the linear relationships between the parameters studied. The level of statistical significance was set at $p<0.05$. Unless otherwise mentioned, all data are presented as mean $\pm \mathrm{SD}$.

\section{Results}

\subsection{Military training and DLW measurement}

Accomplishment of activity measurement. Two of the subjects had the AR data collected from $3 \mathrm{~d}$ only, and thus they were excluded from the analysis of the $7 \mathrm{~d}$ period. The remaining 


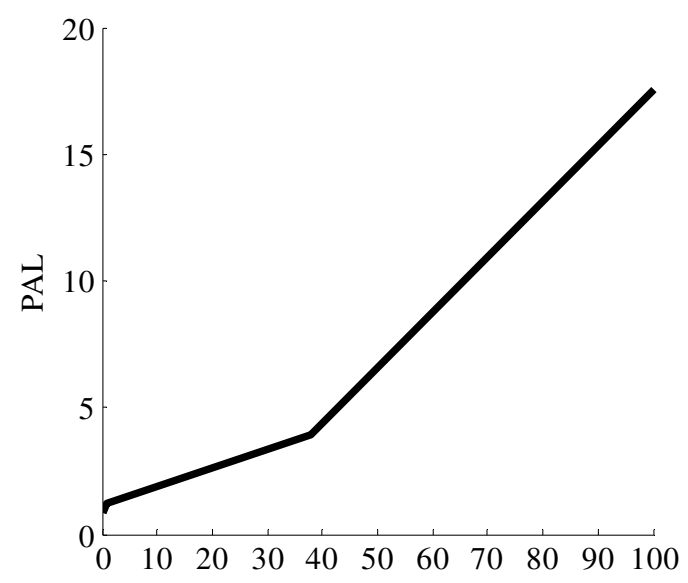

Frequency of hand movements (movements/min)

Figure 1. Customized four-point linear model between the frequency of hand movements and physical activity level (PAL, solid line) in $7 \mathrm{~d}$ of military training $(N=22)$.

Table 2. EE measured by the AR and DLW, $N=22$. With the AR, a customized model predicted PAL using frequency of hand movements, $\mathrm{BH}$, and $\mathrm{BM}$.

\begin{tabular}{llllllr}
\hline & AR & DLW & $t$-test & $r(p)$ & SEE & SEE $(\%)$ \\
\hline PAL & $2.17 \pm 0.19$ & $2.17 \pm 0.23$ & 0.984 & $0.80(<0.001)$ & 0.136 & $6.3 \%$ \\
TEE $\left(\mathrm{MJ} \mathrm{d}^{-1}\right)$ & $16.6 \pm 1.80$ & $16.6 \pm 2.1$ & 0.991 & $0.86(<0.001)$ & 1.05 & $6.3 \%$ \\
AEE $/ \mathrm{BM}\left(\mathrm{kJ} \mathrm{kg}^{-1} \mathrm{~d}^{-1}\right)$ & $98.3 \pm 19.9$ & $98.1 \pm 23.5$ & 0.977 & $0.84(<0.001)$ & 12.5 & $12.8 \%$ \\
\hline
\end{tabular}

SEE: standard error of estimate. SEE (\%): standard error of estimate in per cents. PAL: physical activity level (multiple of BMR). TEE: total energy expenditure $\left(\mathrm{MJ} \mathrm{d}^{-1}\right)$. AEE/BM: activity energy expenditure adjusted with body mass $\left(\mathrm{kJ} \mathrm{kg}^{-1} \mathrm{~d}^{-1}\right)$.

22 subjects' activity measurements were accomplished successfully, and the percentage of measured time share was similar during the days and at night $(80 \% \pm 19 \%$ and $78 \% \pm 12 \%$, respectively, $p=0.499$ ). When comparing $24 \mathrm{~h}$ periods with each other, the measured time share varied between $59 \%$ and $96 \%$ and was not associated with average PAL $\mathrm{PR}_{\mathrm{AR}}$. Between the subjects, the measured time varied from $51 \%$ to $97 \%$, and it was not associated with the individual differences between AR and DLW in PAL, TEE, or AEE/BM.

Customized model between the AR and PAL. The four-point piece-wise linear model that yielded the best fit between $\mathrm{TEE}_{\mathrm{AR}}$ and $\mathrm{TEE}_{\mathrm{DLW}}$ is presented in figure 1. The best $\mathrm{BH}$ adjustment factor was $1.00 \%$ of the minute-by-minute PAL above resting value per $1 \mathrm{~cm}$ difference from the group mean $\mathrm{BH}$, and the $\mathrm{BM}$ adjustment factor was $-0.65 \%$ of the minute-by-minute PAL above resting value per $1 \mathrm{~kg}$ difference from the group mean BM.

Figure 2 shows physical activity graphs over the $7 \mathrm{~d}$ measurement period derived from the $\mathrm{AR}$ and anthropometric parameters from two different subjects. During the $7 \mathrm{~d}$ measurement period, no statistical differences were found in PAL, TEE, and AEE/BM measured with the AR and DLW techniques (table 2, figure 3). A Bland-Altman plot of TEE and AEE/BM showed no significant trend between the mean and the difference of the two methods, and the $95 \%$ confidence intervals were $-2.1-2.1 \mathrm{MJ} \mathrm{d}^{-1}$ in TEE and $-25.1-25.2 \mathrm{~kJ} \mathrm{~kg}^{-1} \mathrm{~d}^{-1}$ in AEE/BM (figure 4). 


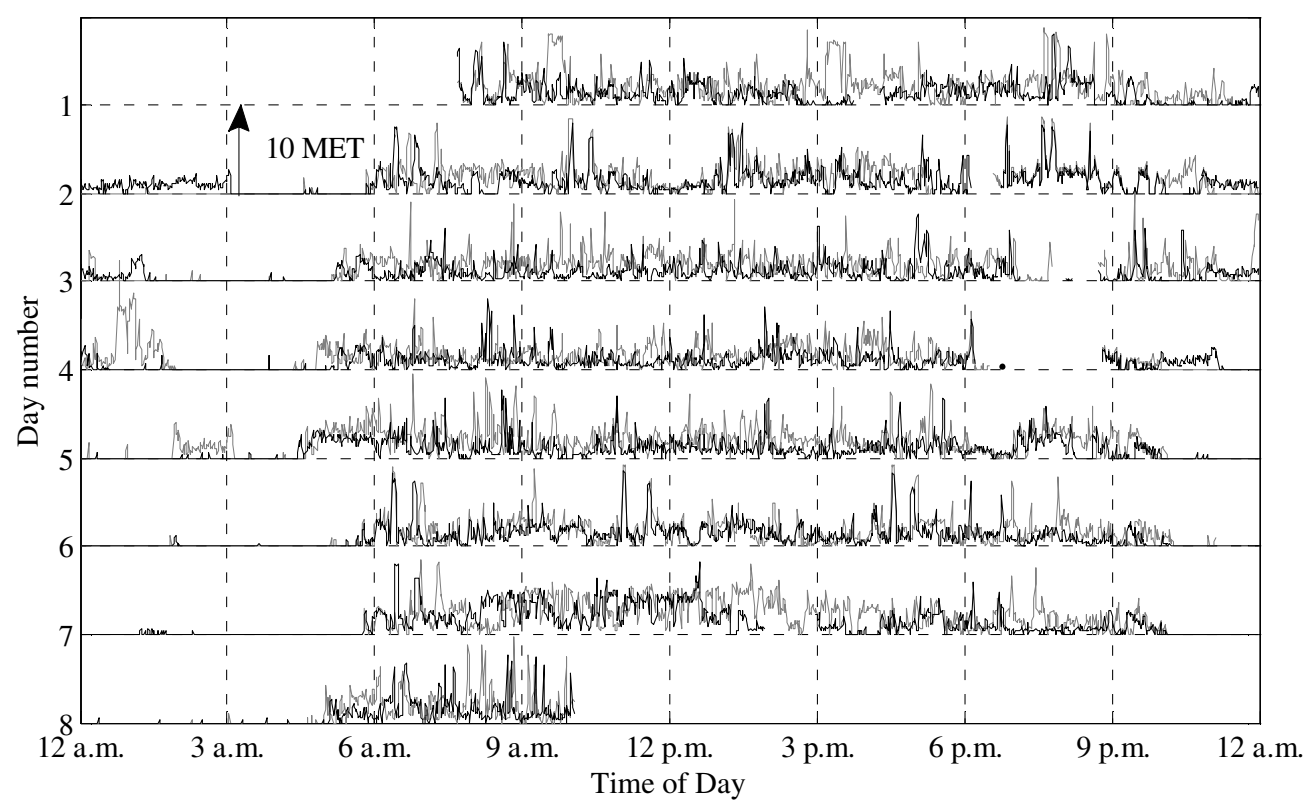

Figure 2. Physical activity of two example subjects measured by the AR during 1 week of military training. The average PAL of the two subjects was 2.41 and 1.90 (gray and black, respectively). The dashed horizontal lines for each day represent full rest and the gap between successive days is scaled to 10 metabolic equivalents. Subject in gray: BM $87.4 \mathrm{~kg}, \mathrm{BH} 187 \mathrm{~cm}, f \_$hand 14.0 mov. $\min ^{-1}$ (12.3 n.u.), TEE $20.3 \mathrm{MJ} \mathrm{d}^{-1}$. Subject in black: BM $98.8 \mathrm{~kg}, \mathrm{BH} 183 \mathrm{~cm}$, $f$ _hand 11.0 mov. min $^{-1}$ (10.0 n.u.), TEE $17.4 \mathrm{MJ} \mathrm{d}^{-1}$. $f$ hand: average frequency of hand, n.u.: nonlinearity normalized units, TEE: total energy expenditure.
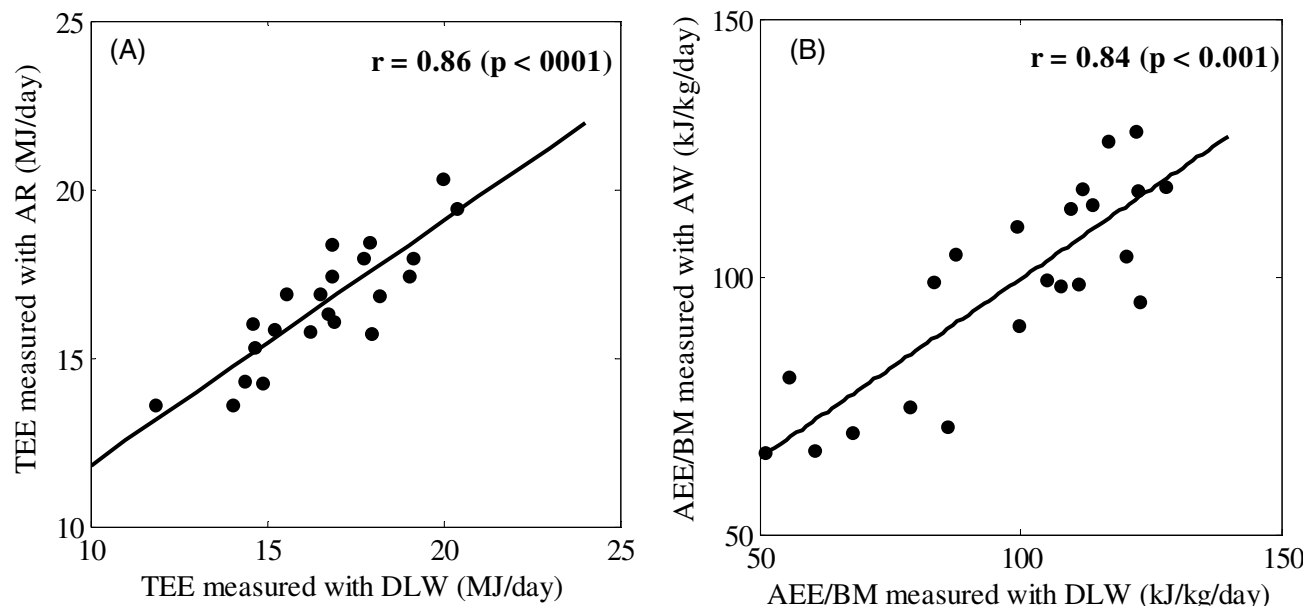

Figure 3. Total energy expenditure (TEE, (A)) and activity-induced energy expenditure adjusted for body mass (AEE/BM, (B)) measured by the AR and DLW technique over the $7 \mathrm{~d}$ period of military training $(N=22)$.

Linear regression models. AR-measured $f$ hand_ $n_{\mathrm{AR}}$ explained $46 \%$ of the variation in

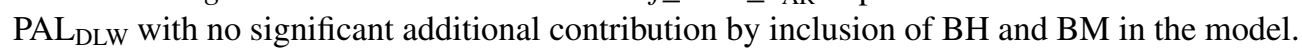
In the TEE model, BM explained $29 \%$ of the variation in $\mathrm{TEE}_{\mathrm{DLW}}$ with a significant added contribution by inclusion of $f \_$hand $\_n_{\mathrm{AR}}(58 \%)$ and $\mathrm{BH}(70 \%)$. Regarding AEE/BM $\mathrm{DLW}_{\mathrm{DL}}$, 

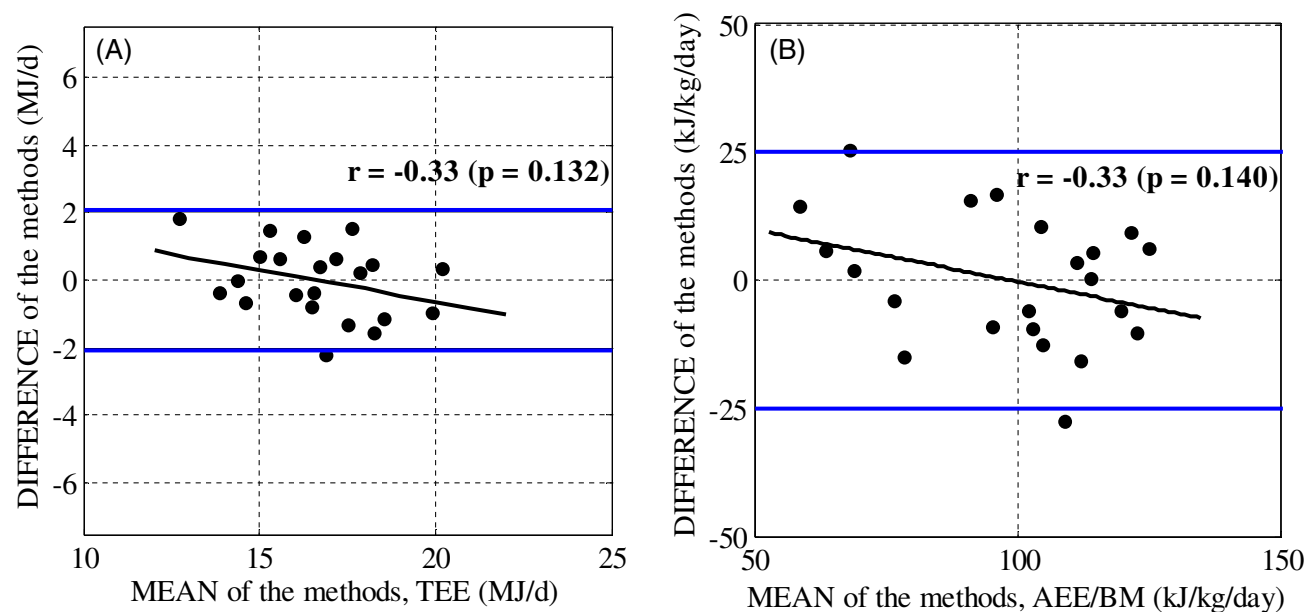

Figure 4. Bland-Altman plots of total energy expenditure (TEE, (A)) and activity energy expenditure adjusted for body mass (AEE/BM, (B)) measured by the AR and DLW technique over the $7 \mathrm{~d}$ period of military training $(N=22)$.

Table 3. Multivariate regression models for explaining the variation in EE parameters $(N=22)$.

\begin{tabular}{|c|c|c|c|c|c|}
\hline Prediction equation & Adjusted $r^{2}$ & $r$ & $p$ & SEE & $\overline{\operatorname{SEE}(\%)}$ \\
\hline $\mathrm{PAL}_{\mathrm{DLW}}=0.153 * f \_$hand $\_n_{\mathrm{AR}}+0.531$ & 0.46 & 0.69 & $<0.001$ & 0.172 & 7.9 \\
\hline $\begin{array}{l}\mathrm{TEE}_{\mathrm{DLW}}=0.064 * \mathrm{BM}+1.25 * f_{-} \text {hand_ } n_{\mathrm{AR}}+ \\
0.120 * \mathrm{BH}-22.9\end{array}$ & 0.70 & 0.86 & $<0.001$ & 1.168 & 7.0 \\
\hline $\begin{array}{l}\mathrm{AEE} / \mathrm{BM}_{\mathrm{DLW}}=15.2 * f_{\perp} \text { hand_ } n_{\mathrm{AR}}- \\
1.01 * \mathrm{BM}+1.30 * \mathrm{BH}-213\end{array}$ & 0.70 & 0.86 & $<0.001$ & 12.96 & 13.2 \\
\hline
\end{tabular}

SEE: standard error of estimate. SEE (\%): standard error of estimate in per cents. PAL ${ }_{\text {DLW: }}$ physical activity level (multiple of BMR). TEE $E_{\text {DLW: }}$ total energy expenditure measured with DLW $\left(\mathrm{MJ} \mathrm{d}^{-1}\right)$. DLW: double labeled water.

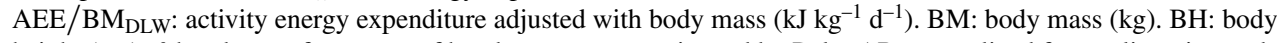
height $(\mathrm{cm}) . f$ hand $\_n_{\mathrm{AR}}$ : frequency of hand movements registered by Polar AR, normalized for nonlinearity, and averaged over the $7 \mathrm{~d}$ period.

$f \_$hand $n_{\mathrm{AR}}$ explained $41 \%$ of the variation with a significant added contribution by inclusion of BM (59\%) and BH (70\%). The prediction equations are shown in table 3.

\subsection{Treadmill test}

All 24 subjects performed the treadmill test and reached the criteria set for maximal effort. The subjects were able to walk and run $5.3 \pm 1.1$ three-minute stages on the treadmill (range 3-7). In 2 subjects the AR apparently failed to detect all steps at the lowest walking speed $\left(<45\right.$ counts $\min ^{-1}$ at $4.6 \mathrm{~km} \mathrm{~h}^{-1}$ ); they were excluded from the analysis. On an individual level, measured $f \_$hand ${ }_{\mathrm{AR}}$ correlated strongly with $\mathrm{PAL}_{\mathrm{IC}}, r=0.97 \pm 0.2$ (range 0.93-1.00). There was, however, some individual variation in the relationship between $f_{-}$hand $\mathrm{AR}_{\mathrm{R}}$ and intensity of exercise (figure 5).

During walking $\left(f \_\right.$hand $\mathrm{AR}_{\mathrm{A}}=60 \mathrm{mov}$. $\mathrm{min}^{-1}$, corresponds to 120 steps $\min ^{-1}$ and about $6 \mathrm{~km} \mathrm{~h}^{-1}$ ) and running ( $80 \mathrm{mov} \cdot \mathrm{min}^{-1}$ and about $\left.11 \mathrm{~km} \mathrm{~h}^{-1}\right)$, BH correlated with $\mathrm{PAL}_{\mathrm{IC}}(r=$ $0.60, p=0.002$ and $r=0.65, p=0.001$, respectively). At the mentioned levels of $f$ hand ${ }_{\mathrm{AR}}$, a $1 \mathrm{~cm}$ change in $\mathrm{BH}$ increased $\mathrm{PAL}_{\mathrm{IC}}$ by $1.3 \%$ in walking and $1.1 \%$ in running. 


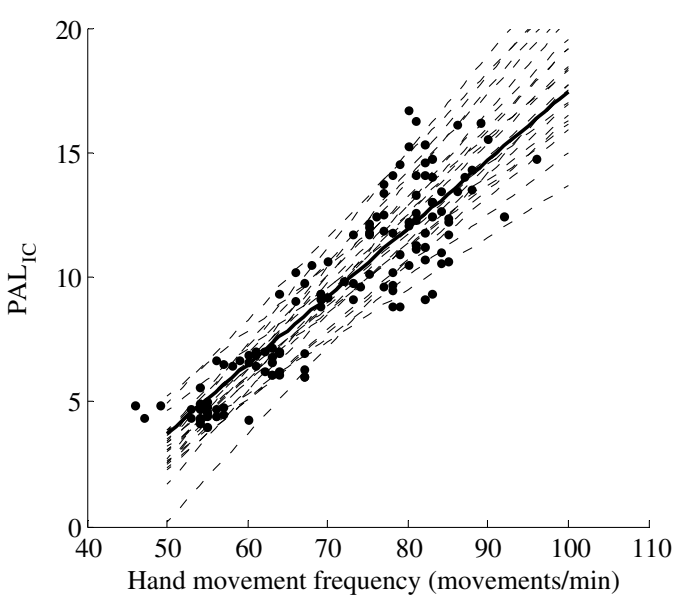

Figure 5. Hand movement frequency registered by a Polar AR and PAL measured by an indirect calorimeter $\left(\mathrm{PAL}_{\mathrm{IC}}\right)$ during treadmill walking and running $(N=24)$. The average individual coefficient of correlation was $0.97 \pm 0.02$ (dashed lines), and for combined data the coefficient of correlation was 0.90 (solid line).

\subsection{Field march test}

From a total of 24 subjects, 4 subjects did not participate in the field march test because of being sick. On an individual level, $f \_$hand ${ }_{\mathrm{AR}}$ was very constant during the $45 \mathrm{~min}$ march (SD $2.3 \pm 1.0$ movements $\min ^{-1}$ ). The average $f$ hand ${ }_{\mathrm{AR}}$ of marching was not different from $f_{-}$hand $\mathrm{AR}_{\mathrm{R}}$ measured in treadmill walking or running at the same speed $(p=0.256)$, and the frequencies correlated with each other $(r=0.77, p<0.001)$. Bland-Altman plot showed no trend between the mean and the difference of the two methods $(r=0.03, p=0.916)$, and the $95 \%$ confidence intervals were $-7.4-5.6$ movements $\min ^{-1}$.

\section{Discussion}

The main focus of this study was to investigate the reliability and validity of the new and practical AR method during military training. During the $7 \mathrm{~d}$ measurement period, AR-derived parameters of EE were compared with those measured with the DLW technique, and a very low standard error of estimate (SEE) and a high positive correlation were found. A Bland-Altman plot showed no trend between the mean and difference of the two methods, indicating that the accuracy of the AR was equal independent of the level of EE. In the treadmill exercise, hand movement frequency correlated highly and positively with $\mathrm{PAL}_{\mathrm{IC}}$, and the correlation improved further by inclusion of $\mathrm{BH}$ in a regression model. In the field marching test, the AR measured similar hand movement frequencies as on the treadmill with the same speeds.

In the signal processing, the main outcome of this research was the determination of a relationship between $f \_$hand $\mathrm{AR}_{\mathrm{AR}}$ and $\mathrm{PAL}_{\mathrm{DLW}}$ which may provide a reliable and robust method for estimating the EE of military training. This was accomplished iteratively by searching for a best fit between the estimated $7 \mathrm{~d}$ TEE and the corresponding value measured with the DLW technique. Furthermore, during the $45 \mathrm{~min}$ march on a flat surface with $20 \mathrm{~kg}$ of military equipment, similar $f_{-}$hand $\mathrm{AR}_{\mathrm{AR}}$ was measured as on the treadmill at the same speed. Comparison between the march and treadmill tests needs to be interpreted with caution because subjects had freedom to select between walk and run in both tests, and we did not account for the 
apparent nonlinearity in the relationship between speed and stride frequency that takes place in transition from walk to run. However, the fact that individual variability in $f_{-}$hand $\mathrm{AR}_{\mathrm{AR}}$ was very low during marching confirmed that the AR was able to register stride frequency of walking or running correctly even though the hands were not able to move freely during the field march test - the subjects held the rifle in front of them. If the speed is maintained, carrying $20 \mathrm{~kg}$ of extra weight increases metabolic requirements significantly (Bilzon et al 2001). Altogether, these results indicate that the AR did not account for the extra amount of load carried. This is an expected finding because accelerometers are, in general, insensitive to extra load carried. This implies that the accuracy of the AR might be improved further by including the amount of extra weight as supplemental data input into the AR.

Use of a wrist-worn accelerometer was shown to be a practical approach in monitoring military training even though the $7 \mathrm{~d}$ period included high-intensity activities under extreme winter conditions. The subjects had no need to remove the device at any time except for data download, and they were able to wear it during days and nights. Some activity data were missed because of difficulties in organizing data downloading during overnight field training and due to sick leave. Nevertheless, activity data were successfully recorded $79 \%$ of the time, which can be considered high enough for reliable comparison of the EE measures of the AR and DLW techniques. A similar percentage of data $(8.2$ out of $10 \mathrm{~d})$ was recently measured in the only study on a wrist-worn device that is available for comparison (van Hees et al 2011).

Comparison of the results obtained from the $7 \mathrm{~d}$ military training period and the treadmill exercise indicates that at the same $f_{-}$hand $_{\mathrm{AR}}$, the intensity of physical activity was higher during the $7 \mathrm{~d}$ period than in the treadmill test which included walking and running. The magnitude of the difference was largest at movement frequencies typical of normal walking. As discussed above concerning the 45 min march test, part of the difference can be attributed to the insensitivity of the accelerometers to extra load carried during military service. Different modes of activities (skiing, fighting) and terrain factors (snow, hills, etc) included in military training may also induce higher energy requirements at the same movement frequencies. The $7 \mathrm{~d}$ military service included cross-country skiing during several days. In skiing data measured by Vähäsöyrinki et al (2008), the poling frequency was 43 cycles $\min ^{-1}$ at slow speed and 61 cycles $\min ^{-1}$ at maximal skiing speed, which represent a smaller range and lower maximal value than in walking and running.

The presented approach to assessing human EE-a wrist-worn 1D accelerometer and a special movement counter-is a most simple and practical way to monitor activity and can be seen as a successor to traditional wrist-worn motion sensors (Montoye et al 1983). The current study is the first one which has compared exercise rate or hand movement frequency with DLW. Van Hees et al (2011) recently compared a wrist-worn 3D accelerometer with DLW by utilizing a more complex signal processing method, and found a significant but clearly smaller contribution of wrist acceleration to AEE (24\%) than what was found in the present study. The partly divergent results obtained therefore deserve methodological discussion.

It is clear that movements of the hands only cause a fraction of AEE. However, in this study $f_{-}$hand ${ }_{\mathrm{AR}}$ strongly predicted $\mathrm{PAL}_{\mathrm{DLW}}$ measured over a $7 \mathrm{~d}$ period and PAL $_{\mathrm{IC}}$ measured during treadmill walking and running. In walking and running, the strong prediction can be explained by the linkage between hand movement frequency and walking cadence, which was also confirmed in this study. Spontaneous choice of running cadence has been associated with optimization of running economy (Cavanagh and Williams 1982), a fact that apparently stabilizes hand movement frequency during walking and running and makes measurement of hand movement frequency more reliable. A wrist-worn accelerometer may underestimate TEE in high-intensity activities, but these usually cover only short periods of time over the whole day and therefore do not contribute strongly to TEE (Westerterp and Plasqui 2004). If 
wrist-worn accelerometers are used to measure the intensity of structured exercise sessions, more complex signal processing methods, including activity classification, may certainly improve prediction accuracy (Siirtola et al 2009). In activities including standing and slow motion, hand movements have a role in maintaining the balance of the body and subsequently may also reflect the whole body's activity level. It is also possible that behavioral aspects, such as the number of restless hand movements within sedentary and standing activities, are also reflected in both estimated and measured TEE. The independent role of the regularity of hand movements, which affects the determination of $f \_$hand ${ }_{\mathrm{AR}}$ (Kinnunen and Miettinen 2005), was not examined in the present study; nevertheless, use of regularity may prevent overestimation of light-intensity activities, because repetitive hand movements are ignored within a longer time window in the case of an irregular movement pattern.

Different parameters of TEE are supposed to strongly depend on each other: TEE = $\mathrm{PAL} * \mathrm{BMR}, \mathrm{AEE} / \mathrm{BM}=(0.9 * \mathrm{TEE}-\mathrm{BMR}) / \mathrm{BM}$, and $\mathrm{BMR}=15.1 * \mathrm{BM}+692$. Nevertheless, the stepwise multivariate linear regression analysis illustrated different contributions of accelerometer data, $\mathrm{BH}$, and BM for each dependent parameter. A reader might wonder why BM was included as a predictor of AEE/BM. Inclusion was considered reasonable because BM may affect not only AEE, but also the relationship between exercise rate and intensity of exercise. In the customized piece-wise linear model designed to predict PAL $\mathrm{DLW}_{\text {, }}$ we decided to include $\mathrm{BH}$ and $\mathrm{BM}$ as correcting factors even though the corresponding multivariate linear regression model was not significantly improved by inclusion of the body parameters. The use of the anthropometric parameters can be justified with both mechanical considerations and a desire to be systematic. It is apparent that mechanical gait characteristics, including step length and cadence, depend on body dimensions (Wixted et al 2007). Systematically thinking, one cannot affect AEE without affecting PAL and TEE-unless there is a compensating change in BMR. Another advantage of our customized equation is that it can also be applied to short periods of time. In addition, the method can be developed further in different groups of people and different activities in the future, even if the DLW technique is not available. Using the customized model, $\mathrm{TEE}_{\mathrm{AR}}$ and $\mathrm{AEE} / \mathrm{BM}_{\mathrm{AR}}$ were derived systematically from PAL $\mathrm{AR}_{\mathrm{A}}$ and estimated BMR, and the accuracy compared well with the separate multivariate regression models.

Measurement of EE during an intensive training week of basic military training would be challenging for any wearable monitor technology and post-processing method. Regardless of the challenges, the magnitude of correlation between $\mathrm{AEE} / \mathrm{BM}_{\mathrm{AR}}$ and $\mathrm{AEE} / \mathrm{BM} \mathrm{DLW}_{\mathrm{DW}}$ $(r=0.86)$ and the contribution of wrist accelerometer measures alone to AEE/BM $\mathrm{BLW}_{\mathrm{DLW}}(41 \%)$ and PAL $\mathrm{PLW}_{\mathrm{D}}(46 \%)$ were among the highest that have been reported in the literature with any accelerometer approaches (Plasqui and Westerterp 2007). However, none of the studies available for comparison have been carried out in military training. The most straightforward applications of this study include estimation of PAL and TEE in soldiers and athletes in order to give feedback on training volume. The average $7 \mathrm{~d}$ activity level $\left(\mathrm{PAL}_{\mathrm{DLW}}=\right.$ 2.17) indicated a very high training volume that is comparable to that of endurance athletes. In conclusion, compared to the DLW technique, the wrist-worn AR method estimated TEE accurately with SEE of $6.3 \%$. Thus the present method can be regarded as a reliable and valid way to assess EE during intensive training.

\section{Acknowledgments}

The authors thank the conscripts who participated in this study. The study was funded by the Finnish Ministry of Education, the Finnish Cultural Foundation, Polar Electro Oy, and the Scientific Advisory Board for Defense. 


\section{References}

Ainsworth B E, Haskell W L, Leon A S, Jacobs D R Jr, Montoye H J, Sallis J F and Paffenbarger R S Jr 1993 Compendium of physical activities: classification of energy costs of human physical activities Med. Sci. Sports Exerc. 25 71-80

ASCM 2001 ASCM'S Guidelines for Exercise Testing and Prescription 6th edn (Baltimore: Lippincott Williams \& Wilkins) pp 5-6, 57, 68, 117, 303

Bilzon J L, Allsopp A J and Tipton M J 2001 Assessment of physical fitness for occupations encompassing loadcarriage tasks Occup. Med. 51 357-61

Black A E, Coward W A, Cole T J and Prentice A M 1996 Human energy expenditure in affluent societies: an analysis of 574 doubly-labelled water measurements Eur. J. Clin. Nutr. $5072-92$

Black A E, Prentice A M and Coward W A 1986 Use of food quotients to predict respiratory quotients for the doubly-labelled water method of measuring energy expenditure Hum. Nutr. Clin. Nutr. 40 381-91

Bland J M and Altman D G 1986 Statistical methods for assessing agreement between two methods of clinical measurement Lancet 1 307-10

Bouten C V, Sauren A A, Verduin M and Janssen J D 1997 Effects of placement and orientation of body-fixed accelerometers on the assessment of energy expenditure during walking Med. Biol. Eng. Comput. 35 50-6

Bouten C V, Westerterp K R, Verduin M and Janssen J D 1994 Assessment of energy expenditure for physical activity using a triaxial accelerometer Med. Sci. Sports Exerc. 26 1516-23

Brugniaux J V, Niva A, Pulkkinen I, Laukkanen R M, Richalet J P and Pichon A P 2010 Polar Activity Watch 200: a new device to accurately assess energy expenditure Br. J. Sports Med. 44 245-9

Cavanagh P R and Williams K R 1982 The effect of stride length variation on oxygen uptake during distance running Med. Sci. Sports Exerc. 14 30-5

Cheng T M, Savkin A V, Celler B G, Su S W and Wang N 2009 Universal algorithm for exercise rate estimation in walking, cycling and rowing using triaxial accelerometry Electron. Lett. 45 394-5

Deurenberg P, Weststrate J A and Seidell J C 1991 Body mass index as a measure of body fatness: age- and sex-specific prediction formulas Br. J. Nutr. 65 105-14

Ekelund U, Yngve A, Brage S, Westerterp K and Sjostrom M 2004 Body movement and physical activity energy expenditure in children and adolescents: how to adjust for differences in body size and age Am. J. Clin. Nutr. $79851-6$

Kinnunen H and Miettinen J 2005 Arrangement, method and computer program for determining physical activity level of human being European Patent No 1532924

Kinnunen H O, Niva A J, Nauha L A and Miettinen J M 2009 Estimation of energy expenditure from wrist attached accelerometer signal using movement counting and filtering Med. Sci. Sports Exerc. 41 (Suppl. 5) 1091

Lusk G 1924 Animal calorimetry. Analysis of the oxidation of mixtures of carbohydrate and fat. A correction J. Biol. Chem. 59 41-2

Montoye H J, Washburn R, Servais S, Ertl A, Webster J G and Nagle F J 1983 Estimation of energy expenditure by a portable accelerometer Med. Sci. Sports Exerc. 15 403-7

Plasqui G and Westerterp K R 2007 Physical activity assessment with accelerometers: an evaluation against doubly labeled water Obesity 15 2371-9

Poehlman E T 1989 A review: exercise and its influence on resting energy metabolism in man Med. Sci. Sports Exerc. 21 515-25

Santtila M, Kyrölainen H, Vasankari T, Tiainen S, Palvalin K, Häkkinen A and Häkkinen K 2006 Physical fitness profiles in young Finnish men during the years 1975-2004 Med. Sci. Sports Exerc. 38 1990-4

Schoeller D A, Ravussin E, Schutz Y, Acheson K J, Baertschi P and Jequier E 1986 Energy expenditure by doubly labeled water: validation in humans and proposed calculation Am. J. Physiol. 250 R823-30

Schofield W N 1985 Predicting basal metabolic rate, new standards and review of previous work Hum. Nutr. Clin. Nutr. 39 5-41

Siirtola P, Laurinen P, Haapalainen E, Röning J and Kinnunen H 2009 Clustering-based activity classification with a wrist-worn accelerometer using basic features IEEE Symp. on Computational Intelligence and Data Mining 2009 CIDM '09 pp 95-100

Speakman J R and Westerterp KR 2010 Associations between energy demands, physical activity, and body composition in adult humans between 19 and 96 y of age Am. J. Clin. Nutr. 92 826-34

Swartz A M, Strath S J, Bassett D R Jr, O'Brien W L, King G A and Ainsworth B E 2000 Estimation of energy expenditure using CSA accelerometers at hip and wrist sites Med. Sci. Sports Exerc. 32 S450-6

Tanaka C, Tanaka S, Kawahara J and Midorikawa T 2007 Triaxial accelerometry for assessment of physical activity in young children Obesity 15 1233-41 
Tanskanen M, Uusitalo A L, Häkkinen K, Nissilä J, Santtila M, Westerterp K R and Kyröläinen H 2009 Aerobic fitness, energy balance, and body mass index are associated with training load assessed by activity energy expenditure Scand. J. Med. Sci. Sports 19 871-8

Tudor-Locke C and Rowe D A 2012 Using cadence to study free-living ambulatory behaviour Sports Med. 42 381-98

Vähäsöyrinki P, Komi P V, Seppala S, Ishikawa M, Kolehmainen V, Salmi J and Linnamo V 2008 Effect of skiing speed on ski and pole forces in cross-country skiing Med. Sci. Sports Exerc. 40 1111-6

van Hees V T et al 2011 Estimation of daily energy expenditure in pregnant and non-pregnant women using a wrist-worn tri-axial accelerometer PLoS One 6 e22922

Wagenaar R C and van Emmerik R E A 2000 Resonant frequencies of arms and legs identify different walking patterns J. Biomech. $33853-61$

Westerterp K R 1998 Alterations in energy balance with exercise Am. J. Clin. Nutr. 68 970S-4S

Westerterp K R and Plasqui G 2004 Physical activity and human energy expenditure Curr. Opin. Clin. Nutr. Metab. Care 7 607-13

Westerterp K R and Speakman J R 2008 Physical activity energy expenditure has not declined since the 1980s and matches energy expenditures of wild mammals Int. J. Obes. 32 1256-63

Westerterp K R, Wouters L and van Marken Lichtenbelt W D 1995 The Maastricht protocol for the measurement of body composition and energy expenditure with labeled water Obes. Res. $349-57$

Wixted A J, Thiel D V, Hahn A G, Gore C J, Pyne D B and James D A 2007 Measurement of energy expenditure in elite athletes using MEMS-based triaxial accelerometers IEEE Sensors J. 7 481-8

Yang C C and Hsu Y L 2010 A review of accelerometry-based wearable motion detectors for physical activity monitoring Sensors $107772-88$

Zhang S, Rowlands A V, Murray P and Hurst T 2012 Physical activity classification using the GENEA wrist-worn accelerometer Med. Sci. Sports Exerc. 44 742-8 